\title{
Características estruturais de bosques de mangue do estuário do rio São Mateus, ES, Brasil
}

\author{
Maria Amélia Bonfante da Silva ${ }^{1}$, Elaine Bernini ${ }^{1,3,4}$ e Tania Mara Simões do Carmo ${ }^{2}$
}

Recebido em 14/02/2003. Aceito em 18/11/2004

\begin{abstract}
RESUMO - (Características estruturais de bosques de mangue do estuário do rio São Mateus, ES, Brasil). A estrutura da vegetação do manguezal do estuário do rio São Mateus foi analisada pelo método de parcelas em quatro sítios de estudo. Nesse manguezal, foram registradas as espécies Avicennia germinans (L.) Stearn., Avicennia schaueriana Stapft \& Leechm., Laguncularia racemosa (L.) Gaertn. e Rhizophora mangle L. Considerando todas as parcelas, o DAP médio variou de 8,12 a 29,6 cm, a altura média de 5,4 a 12,0 $\mathrm{m}$, a área basal de 7,21 a $31,1 \mathrm{~m}^{2}$ ha $^{-1}$ e a densidade de 450 a 1.450 ind.ha $^{-1}$. Os sítios de estudo sob maior influência das marés apresentaram menor desenvolvimento estrutural e dominância de $R$. mangle, enquanto que o melhor desenvolvimento estrutural, com dominância de L. racemosa e A. germinans, foi observado nos sítios sujeitos ao maior aporte de água doce. Os resultados demonstraram que o manguezal do estuário do rio São Mateus apresenta bom desenvolvimento estrutural em relação a outros manguezais do Estado do Espírito Santo.
\end{abstract}

Palavras-chave: manguezal, estrutura da vegetação, estuário do Rio São Mateus

\begin{abstract}
Structural characteristics of the mangrove forests at São Mateus River Estuary, Espírito Santo State, Brazil). The structure of the mangrove forests at São Mateus River Estuary was analyzed using the plot method. Mangrove species at the estuary are Avicennia germinans (L.) Stearn., Avicennia schaueriana Stapft \& Leechm., Laguncularia racemosa (L.) Gaertn., and Rhizophora mangle L. Structural analyzes were performed in four sites. In all stands, the DBH varied from 8.12 to $29.6 \mathrm{~cm}$, average height from 5.4 to $12.0 \mathrm{~m}$, the basal area from 7.21 to $31.1 \mathrm{~m}^{2} . \mathrm{ha}^{-1}$, and the density from 450 a 1,450 ind.ha ${ }^{-1}$. Sites under larger influence of the tides, presented smaller structural development and dominance of $R$. mangle. L. racemosa and A. germinans were dominant in sites with fresh water predominance. The results obtained demonstrated that mangrove which was studied presented a good structural development in relation to other mangroves located in the Espírito Santo State.
\end{abstract}

Key words: mangrove, structure forest, São Mateus River Estuary

\section{Introdução}

O manguezal é um ecossistema costeiro de transição entre os ambientes terrestre e marinho, característico de regiões tropicais e subtropicais e sujeito ao regime das marés. Ocorre em regiões costeiras abrigadas como estuários, baías e lagunas, e apresenta condições propícias para alimentação, proteção e reprodução para muitas espécies animais, sendo considerado importante transformador de nutrientes em matéria orgânica e gerador de bens e serviços (Schaeffer-Novelli 1995).

Segundo Walsh (1974), o melhor grau de desenvolvimento do manguezal dependeria de cinco requisitos: (1) temperaturas tropicais, com temperatura média do mês mais frio superior a $20^{\circ} \mathrm{C}$ (entretanto, a amplitude térmica anual não deve exceder a $5^{\circ} \mathrm{C}$ ); (2) substratos predominantemente lodosos, constituídos de silte e argila e alto teor de matéria orgânica; (3) áreas abrigadas, livres da ação de marés fortes; (4) presença de água salgada, pois as plantas de mangue são halófitas facultativas e dependem desse requisito para competir com as glicófitas que não toleram a salinidade; (5) elevada amplitude de marés.

Ao longo da costa brasileira, os manguezais apresentam-se com características estruturais bastante distintas. Para auxiliar a interpretação das florestas de mangue brasileiras, Schaeffer-Novelli et al. (1990) dividiram o litoral do país em oito unidades fisiográficas, levando-se em consideração relevo, tipo de solo,

\footnotetext{
Universidade Federal do Espírito Santo, Departamento de Biologia, Laboratório de Botânica, Pólo Universitário de São Mateus

2 Universidade Federal do Espírito Santo, Departamento de Biologia, Ciências Humanas e Naturais, Laboratório de Bioecologia do Manguezal (tmsdc@uol.com.br)

3 Universidade Estadual do Norte Fluminense, Laboratório de Ciências Ambientais, Centro de Biociências e Biotecnologia, Av. Alberto Lamego 2000, CEP 28013-602, Campos dos Goytacazes, RJ, Brasil

4 Autora para correspondência: bernini@uenf.br
} 
cobertura vegetal, temperatura média anual, evapotranspiração potencial e amplitude de marés. De acordo com essa divisão, cada unidade apresentaria um desenvolvimento estrutural similar, por estar submetida às mesmas condições ambientais regionais.

Distribuindo-se desde o riacho Doce, no extremo norte, até o rio Itabapoana, na divisa com o Estado do Rio de Janeiro, os manguezais do Espírito Santo ocupam área aproximada de $70 \mathrm{~km}^{2} \mathrm{e}$, de acordo com a classificação de Shaeffer-Novelli et al. (1990), estão incluídos na unidade VI, trecho compreendido entre o Cabo de Santo Antônio, no Recôncavo Baiano, BA

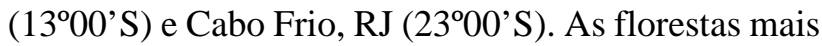
extensas são encontradas no entorno da baía de Vitória e nos estuários dos rios Piraqueaçu e São Mateus (Vale \& Ferreira 1998). A composição florística dos bosques consiste nas espécies Rhizophora mangle L., Laguncularia racemosa (L.) Gaertn., Avicennia schaueriana Stapft \& Leechm. e Avicennia germinans (L.) Stearn., ocorrendo ainda os gêneros Spartina, Hibiscus e Acrostichum, como associados.

As pressões originadas pelo aumento populacional, produção de alimentos e desenvolvimento industrial e urbano têm causado a destruição de significativa parcela dos recursos do manguezal existentes no mundo (Field 1996). No decorrer dos últimos anos, o manguezal do estuário do rio São Mateus, localizado no município de Conceição da Barra, ES, tem sido alvo de freqüentes ações de degradação.

O estuário do rio São Mateus caracteriza-se pela ocorrência de processos flúvio-marinhos que vêm acarretando sérios problemas de erosão na sua principal praia, localizada à margem esquerda, além de grandes alterações fisionômicas nos bosques de mangue de toda sua foz, as quais se devem ora a processos erosivos, ora a processos de acelerada sedimentação. Em função dessas alterações morfológicas, a cidade de Conceição da Barra enfrenta vários problemas sociais e econômicos, relacionados à destruição de dezenas de residências, bem como à dificuldade de navegação de embarcações pesqueiras (C.C. Vale, dados não publicados).

A caracterização estrutural da vegetação do manguezal constitui valiosa ferramenta no que concerne à resposta desse ecossistema às condições ambientais existentes, bem como aos estudos e ações que levam à conservação do ambiente (Soares 1999). $\mathrm{O}$ estudo da estrutura da vegetação analisa o grau de desenvolvimento da floresta, possibilitando a identificação e a delimitação de bosques com características semelhantes (Schaeffer-Novelli \& Cintrón 1986).
O objetivo do presente estudo foi caracterizar a estrutura de bosques de mangue do estuário do rio São Mateus, com o propósito de compará-la com a de outros manguezais do Estado do Espírito Santo e fornecer subsídios à conservação do ecossistema.

\section{Material e métodos}

Área de estudo - A bacia do rio São Mateus localiza-se ao norte do Estado do Espírito Santo, abrangendo área aproximada de $13.500 \mathrm{~km}^{2}$. Os rios Cotoxé e Cricaré têm suas nascentes situadas em Minas Gerais. A confluência desses rios ocorre no baixo curso da bacia, no município de São Mateus, recebendo o nome de rio São Mateus. Ao alcançar os terrenos quaternários, o rio São Mateus direciona-se para o norte, vindo a desaguar no Oceano Atlântico, em Conceição da Barra, ES (C.C. Vale, dados não publicados).

A área de manguezal selecionada para este estudo encontra-se no estuário do rio São Mateus, localizado à sudeste do município de Conceição da Barra (18 35'S; 39 44'W) (Fig. 1). Esse manguezal ocupa área aproximada de $11 \mathrm{~km}^{2}$. No estuário inferior do rio, as espécies vegetais dominantes são $R$. mangle e L. racemosa. A primeira domina a franja dos bosques, enquanto a segunda ocorre mais para o interior, participando da composição de bosques mistos. A ocorrência de A. schaueriana torna-se significativa próxima às áreas de restinga. A espécie A. germinans ocorre, principalmente, nos estuários médio e superior do rio (C.C. Vale, dados não publicados).

No estuário inferior, ocorre lançamento de efluentes domésticos diretamente no rio ou diluídos ao serem misturados ao sistema pluvial. Nos manguezais próximos, verificou-se disposição de lixo e locais que sofreram corte de vegetação, tanto para obtenção de lenha como para ocupações irregulares. As ocupações são feitas principalmente pela população de baixa renda, no entanto, também foi constatada a presença de edificações municipais. Em praticamente todo manguezal, existe a utilização de "redinhas" para captura do caranguejo Ucides cordatus L.

O estuário do rio São Mateus encontra-se sob o regime micromareal, com marés semidiurnas. Baseado em dados do Terminal de Barra do Riacho, ES (1950'S; $40^{\circ} 03^{\prime} \mathrm{W}$ ) no período de 1998 a 2000, a média das marés foi $0,8 \mathrm{~m}$, com intervalo médio entre 0,1 e 1,5 m (Diretoria de Hidrografia e Navegação, Ministério da Marinha). O clima da região é tropical úmido. Considerando a década de 1990, a temperatura média foi $23,9^{\circ} \mathrm{C}$, a média das temperaturas máximas dos 

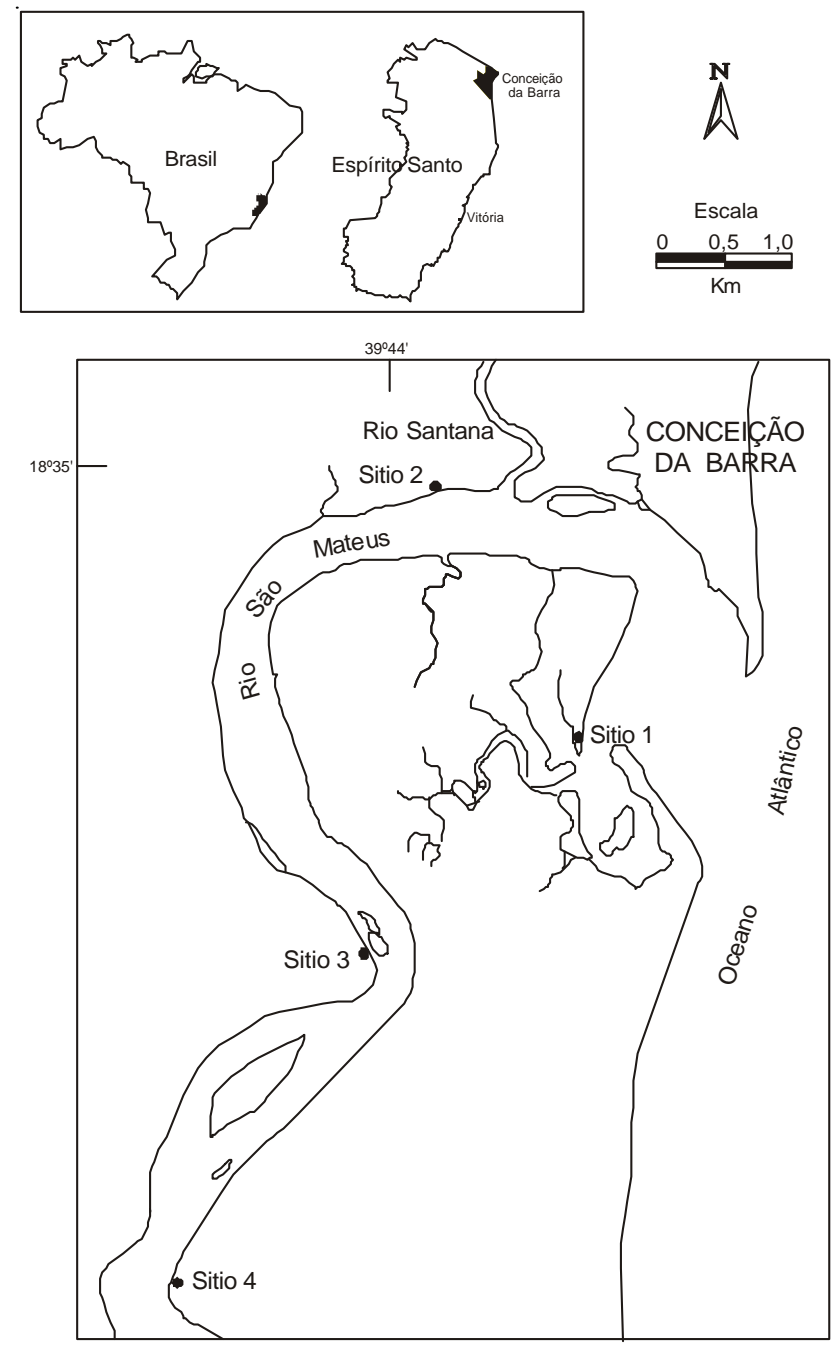

Figura 1. Localização dos sítios de estudo no manguezal do estuário do rio São Mateus, Conceição da Barra, ES, Brasil.

meses mais quentes (janeiro, fevereiro e março) foi $28,9^{\circ} \mathrm{C}$ e a média das mínimas dos meses mais frios (junho, julho e agosto) foi $20,0^{\circ} \mathrm{C}$. A amplitude térmica anual foi de $8,9^{\circ} \mathrm{C}$. A pluviosidade média mensal nesse período variou de 43,2 $\mathrm{mm}$ (junho) a 201,2 $\mathrm{mm}$ (novembro), com o total anual de aproximadamente $1.372 \mathrm{~mm}$ (Fonte dos dados: Instituto Capixaba de Pesquisa, Assistência e Extensão Rural (INCAPER), Linhares, ES - $19^{\circ} 24^{\prime}$ 'S; $40^{\circ} 04^{\prime} \mathrm{W}$ ).

Metodologia - O estudo foi realizado no período de maio/1999 a abril/2000. A caracterização da estrutura vegetal do manguezal foi baseada na metodologia proposta por Schaeffer-Novelli \& Cintrón (1986). A partir de pontos distintos em relação à distância do mar, foram selecionados quatro sítios de estudo, no estuário do rio São Mateus (Fig. 1): Sítio 1 (S-1) - à margem direita do rio, próximo à desembocadura (estuário inferior); Sítio 2 (S-2) - à margem esquerda, próximo ao rio Santana (estuário inferior); Sítio 3 (S-3) - à margem esquerda (estuário médio); Sítio 4 (S-4) à margem direita (estuário médio).

Em cada sítio foi delimitada uma parcela de $20 \times 20 \mathrm{~m}$, a $10 \mathrm{~m}$ da margem do rio. O tamanho da parcela foi definido de modo a garantir a representatividade dos pontos amostrais (Schaeffer-Novelli \& Cintrón 1986). Em cada parcela foram coletadas três amostras de sedimento, a uma profundidade de $20 \mathrm{~cm}$, para análise de granulometria (método de pipetagem) e do percentual de matéria orgânica (digestão úmida com dicromato de potássio e ácido sulfúrico). As análises foram realizadas no Laboratório de Solos do INCAPER, de acordo com métodos descritos em EMBRAPA (1997). As classificações texturais seguiram a metodologia de Shepard (Suguio 1973), baseada nas percentagens de areia, silte e argila. Para efeito de cálculos, as frações areia grossa e areia fina foram somadas, sendo consideradas como fração areia. Em cada parcela, também foram instalados três tubos fixos (30 $\mathrm{cm}$ de profundidade) para determinação do $\mathrm{pH}$ (pHmeter modelo pHTestr 2TM) e da salinidade (refratômetro modelo 10049 American Optical) da água intersticial.

Dentro de cada parcela, os indivíduos com diâmetro à altura do peito $(\mathrm{DAP}) \geq 2,5 \mathrm{~cm}$ foram identificados quanto à espécie e tiveram o diâmetro e a altura total medidos. O DAP foi medido com uma trena calibrada para leitura direta de diâmetro (Ritcher) e a altura, com um telêmetro óptico (Opti-meter Ranging $\left.{ }^{\circledR} 120\right)$.

Posteriormente, foram calculados o DAP médio, a altura média do bosque para cada parcela, as áreas basais viva e morta por espécie e por classe diamétrica, as densidades de indivíduos vivos e mortos para cada espécie, a dominância em área basal e densidade de indivíduos vivos e mortos.

\section{Resultados}

O sedimento apresentou características mais grosseiras no S-1, sendo classificado como arenoso. Nos demais sítios de estudo, as classificações texturais do sedimento variaram de lama síltico-arenosa a lama siltosa. O maior percentual de matéria orgânica foi registrado no Sítio 4 (S-4: 10,9\%) e o menor, no Sítio 2 (S-2: 0,17\%) (Tab. 1).

A salinidade e o pH da água intersticial apresentaram grande variação entre os períodos de coleta e entre os sítios de estudo. Os valores extremos 
Tabela 1. Valores médios $(n=3)$ das frações granulométricas (\%), classificação textural e matéria orgânica (\%) do sedimento analisado no manguezal do estuário do rio São Mateus, ES, Brasil. A = arenoso; LSA = lama síltico arenosa; LS = lama siltosa.

\begin{tabular}{lcccccc}
\hline Sítio & Areia grossa & Areia fina & Silte & Argila & $\begin{array}{c}\text { Classificação } \\
\text { textural }\end{array}$ & $\begin{array}{c}\text { Matéria } \\
\text { orgânica }\end{array}$ \\
\hline S-1 & $24,00 \pm 13,00$ & $72,70 \pm 14,40$ & - & $3,30 \pm 2,31$ & A & 0,17 \\
S-2 & $10,00 \pm 4,36$ & $13,70 \pm 7,23$ & $29,30 \pm 5,51$ & $44,00 \pm 7,21$ & LSA & 1,23 \\
S-3 & $3,50 \pm 0,71$ & $21,00 \pm 9,90$ & $26,50 \pm 2,12$ & $49,00 \pm 12,73$ & LSA & 1,70 \\
S-4 & $7,70 \pm 0,58$ & $9,30 \pm 7,57$ & $29,00 \pm 3,61$ & $54,00 \pm 5,29$ & LS & 10,90 \\
\hline
\end{tabular}

de salinidade oscilaram entre $2(\mathrm{~S}-4)$ e $38(\mathrm{~S}-2)$ e de $\mathrm{pH}$ variaram entre 5,7 (S-4) e 7,9 (S-2) (Tab. 2).

A altura e o DAP médio das árvores tenderam a diminuir no sentido rio-mar, ou seja, do Sítio 4 para o Sítio 1 (Tab. 3). Registrou-se corte de vegetação nos sítios 2 e 3 (Tab. 3). A contribuição em área basal dos indivíduos vivos e mortos com DAP $\geq 10,0 \mathrm{~cm}$ foi maior nos sítios 3 e 4 . Nos sítios localizados no estuário inferior (S-1 e S-2), houve maior contribuição na classe diamétrica entre 2,5 e 10,0 cm (Tab. 4).

Foram verificados valores semelhantes e mais baixos de área basal viva para os sítios localizados no estuário inferior $\left(\mathrm{S}-1: 7,51 \mathrm{~m}^{2} \cdot \mathrm{ha}^{-1}\right.$ e $\mathrm{S}-2$ : $\left.7,21 \mathrm{~m}^{2} \cdot \mathrm{ha}^{-1}\right)$, enquanto que no estuário médio os valores foram mais elevados, com destaque para o Sítio 4 (S-3: 17,0 m².ha ${ }^{-1}$ e S-4: 31,1 m².ha ${ }^{-1}$ ) (Tab. 4). $\mathrm{O}$ mesmo resultado foi observado para área basal morta. A densidade total de indivíduos vivos tendeu a aumentar no sentido rio-mar (450 ind.ha ${ }^{-1}$, no S-4 e 1.450 ind.ha ${ }^{-1}$, no S-1) (Tab. 5).

Nos sítios 1 e 2, a espécie $R$. mangle foi dominante tanto em área basal como em densidade, enquanto no estuário médio, as espécies dominantes foram
L. racemosa (S-3) e A. germinans (S-4). No que diz respeito aos indivíduos mortos, $L$. racemosa apresentou maior dominância em área basal e em número de indivíduos, nos sítios 1,2 e 3 e A. germinans foi dominante no Sítio 4 (Tab. 6).

\section{Discussão}

Os resultados dos parâmetros estruturais obtidos para os quatro sítios de estudo demonstraram diferenças no desenvolvimento estrutural dos bosques de mangue, as quais correspondem às variações da frequiência e da periodicidade das energias subsidiárias, tais como: marés, água doce e aporte de nutrientes. Essas diferenças fisiográficas são definidas por Odum (1967, apud Schaeffer-Novelli et al. 1994) como impressão digital ("finger print") do ecossistema.

Os percentuais das frações inorgânicas e o teor de matéria orgânica do sedimento mostraram-se variáveis, de acordo com a localização dos sítios de estudo, correspondendo às diferenças da hidrodinâmica. As características mais grosseiras do sedimento e os menores percentuais de matéria

Tabela 2. Valores médios $(\mathrm{n}=3)$ de salinidade e pH da água intersticial analisada no manguezal do estuário do rio São Mateus, ES, Brasil.

\begin{tabular}{|c|c|c|c|c|c|c|c|c|}
\hline \multirow[t]{2}{*}{ Data de coleta } & \multicolumn{2}{|c|}{ Sítio 1} & \multicolumn{2}{|c|}{ Sítio 2} & \multicolumn{2}{|c|}{ Sítio 3} & \multicolumn{2}{|c|}{ Sítio 4} \\
\hline & Salinidade & $\mathrm{pH}$ & Salinidade & $\mathrm{pH}$ & Salinidade & $\mathrm{pH}$ & Salinidade & $\mathrm{pH}$ \\
\hline 01/05/99 & 27,5 & 6,9 & 28,0 & 7,5 & 18,5 & 7,2 & 5,0 & 5,9 \\
\hline $16 / 05 / 99$ & 32,0 & 7,0 & 29,5 & 7,4 & 22,0 & 6,7 & 13,0 & 5,8 \\
\hline 13/06/99 & 33,5 & 6,7 & 35,0 & 7,1 & 17,0 & 6,9 & 8,0 & 5,7 \\
\hline 29/06/99 & 31,0 & 7,1 & 36,0 & 6,9 & 16,0 & 7,2 & 15,0 & 6,6 \\
\hline $31 / 07 / 99$ & 33,0 & 7,2 & 32,5 & 7,4 & 15,5 & 7,1 & 8,0 & 5,7 \\
\hline 29/08/99 & 33,0 & 7,2 & 34,5 & 7,3 & 24,5 & 7,1 & 10,0 & 6,1 \\
\hline 28/10/99 & 26,5 & 7,1 & 38,0 & 7,9 & 30,0 & 7,1 & 22,0 & 6,1 \\
\hline 27/12/99 & 25,0 & 6,3 & 30,5 & 7,5 & 10,0 & 6,9 & 6,0 & 6,5 \\
\hline $25 / 01 / 00$ & 21,0 & 6,7 & 22,5 & 7,2 & 9,5 & 6,6 & 3,0 & 5,8 \\
\hline $26 / 02 / 00$ & 16,0 & 7,0 & 18,0 & 7,3 & 7,0 & 6,8 & - & - \\
\hline $31 / 03 / 00$ & 27,5 & - & 10,0 & 6,8 & 3,5 & 7,1 & - & - \\
\hline $30 / 04 / 00$ & 32,0 & - & 17,0 & 7,7 & 5,0 & 7,7 & 2,0 & 7,0 \\
\hline
\end{tabular}


Tabela 3. Número de indivíduos vivos amostrados (N), altura, DAP médio e percentagem de árvores cortadas nos sítios de estudo analisados no manguezal do estuário do rio São Mateus, ES, Brasil.

\begin{tabular}{lccrrc}
\hline Sítio & \multirow{2}{*}{$\mathrm{N}$} & \multicolumn{2}{c}{ Altura $(\mathrm{m})$} & \multirow{2}{*}{ DAP $(\mathrm{cm})$} & $\begin{array}{c}\text { \% de árvores } \\
\text { cortadas }\end{array}$ \\
\cline { 3 - 4 } & & \multicolumn{1}{c}{ Média } & Dossel & & - \\
\hline S-1 & 58 & $5,59 \pm 1,5$ & 8,4 & 8,1 & 11,9 \\
S-2 & 49 & $5,40 \pm 1,8$ & 8,1 & 8,2 & 2,74 \\
S-3 & 44 & $7,92 \pm 1,8$ & 9,9 & 14,0 & - \\
S-4 & 18 & $12,00 \pm 7,0$ & 21,0 & 29,6 & \\
\hline
\end{tabular}

orgânica constatados no Sítio 1 devem-se ao acelerado processo de assoreamento que ocorre na foz do rio São Mateus.

Houve grande variação quanto aos valores de $\mathrm{pH}$ e salinidade da água intersticial. Tais variações refletem as flutuações cíclicas da maré e do aporte de água doce. Os sítios 1 e 2 apresentaram maiores valores de salinidade, por se localizarem no estuário inferior, estando sujeitos a maior influência das marés.

A granulometria do sedimento, provavelmente, não influenciou na estrutura da vegetação, pois as áreas com classificações texturais diferentes ( $\mathrm{S}-1$, arenoso e S-2, lama síltico-arenosa) apresentaram desenvolvimento estrutural semelhante. No entanto, o assoreamento verificado no sítio $\mathrm{S}-1$ pode vir a comprometer a presença de manguezal na área, visto que elevadas taxas de sedimentação interferem na reciclagem dos nutrientes e no intercâmbio dos gases, em decorrência do entupimento das lenticelas de rizóforos e pneumatóforos (Odum \& Johannes 1975). Em áreas assoreadas próximo ao Sítio 1, as plantas de mangue estão sendo substituídas por plantas de restinga, sendo a comunidade de praia constituída principalmente por espécies psamófila-reptantes.

As árvores mais baixas foram registradas nos Sítios 1 e 2, as quais foram associadas com salinidades mais elevadas (S-1: 16,0-33,5 e S-2: 10,0-38,0). Os resultados do presente estudo coincidem com as descrições de Cintrón et al. (1978) que demonstraram a relação entre a altura das árvores de mangue e a salinidade do sedimento.

Considerando a classificação dos tipos fisiográficos de bosque de mangue proposta por Lugo \& Snedaker (1974) e modificada por Cintrón et al.

Tabela 4. Área basal $\left(\mathrm{m}^{2} \cdot \mathrm{ha}^{-1}\right)$ viva e morta por classe diamétrica nos sítios analisados no manguezal do estuário do rio São Mateus, ES, Brasil.

\begin{tabular}{|c|c|c|c|c|c|c|c|}
\hline \multirow[t]{2}{*}{ Sítio } & \multicolumn{3}{|c|}{ Área basal viva } & \multicolumn{3}{|c|}{ Área basal morta } & \multirow[t]{2}{*}{ Total geral } \\
\hline & $\geq 2,5 \mathrm{~cm}$ & $\geq 10,0 \mathrm{~cm}$ & Total & $\geq 2,5 \mathrm{~cm}$ & $\geq 10,0 \mathrm{~cm}$ & Total & \\
\hline S-1 & 3,80 & 3,71 & 7,51 & 0,05 & - & 0,05 & 7,56 \\
\hline S-2 & 5,20 & 2,01 & 7,21 & 0,13 & - & 0,13 & 7,34 \\
\hline S-3 & 3,29 & 13,70 & 17,00 & 0,26 & 10,50 & 10,80 & 27,80 \\
\hline S-4 & 0,60 & 30,50 & 31,10 & 0,80 & 14,20 & 15,00 & 46,10 \\
\hline
\end{tabular}

Tabela 5. Área basal $\left(\mathrm{m}^{2} \cdot \mathrm{ha}^{-1}\right)$ e densidade de indivíduos (ind. ha $\left.{ }^{-1}\right)$ vivos e mortos por espécie nos sítios analisados no manguezal do estuário do rio São Mateus, ES, Brasil.

\begin{tabular}{|c|c|c|c|c|c|c|c|c|c|c|c|}
\hline \multirow[t]{2}{*}{ Sítio } & \multicolumn{5}{|c|}{ Vivos } & \multicolumn{5}{|c|}{ Mortos } & \multirow[t]{2}{*}{ Total geral } \\
\hline & $\mathrm{Ag}$ & As & $\mathrm{Lr}$ & $\mathrm{Rm}$ & Total & $\mathrm{Ag}$ & As & $\mathrm{Lr}$ & $\mathrm{Rm}$ & Total & \\
\hline S-1 & - & $\begin{array}{c}1,26 \\
(150)\end{array}$ & $\begin{array}{c}1,26 \\
(375)\end{array}$ & $\begin{array}{c}4,99 \\
(925)\end{array}$ & $\begin{array}{c}7,51 \\
(1450)\end{array}$ & - & - & $\begin{array}{l}0,05 \\
(25)\end{array}$ & - & $\begin{array}{l}0,05 \\
(25)\end{array}$ & $\begin{array}{c}7,56 \\
(1425)\end{array}$ \\
\hline S-2 & - & $\begin{array}{c}0,43 \\
(225)\end{array}$ & $\begin{array}{l}0,77 \\
(75)\end{array}$ & $\begin{array}{c}6,01 \\
(925)\end{array}$ & $\begin{array}{c}7,21 \\
(1225)\end{array}$ & - & - & $\begin{array}{l}0,10 \\
(50)\end{array}$ & $\begin{array}{l}0,03 \\
(25)\end{array}$ & $\begin{array}{l}0,13 \\
(75)\end{array}$ & $\begin{array}{c}7,34 \\
(1300)\end{array}$ \\
\hline$S-3$ & - & - & $\begin{array}{l}13,40 \\
(900)\end{array}$ & $\begin{array}{c}3,64 \\
(200)\end{array}$ & $\begin{array}{r}17,10 \\
(1100)\end{array}$ & - & - & $\begin{array}{l}10,80 \\
(425)\end{array}$ & - & $\begin{array}{l}10,80 \\
(425)\end{array}$ & $\begin{array}{r}27,80 \\
(1525)\end{array}$ \\
\hline S-4 & $\begin{array}{l}30,10 \\
(325)\end{array}$ & - & $\begin{array}{l}0,60 \\
(50)\end{array}$ & $\begin{array}{l}0,36 \\
(75)\end{array}$ & $\begin{array}{l}31,10 \\
(450)\end{array}$ & $\begin{array}{l}13,80 \\
(350)\end{array}$ & - & $\begin{array}{l}0,24 \\
(50)\end{array}$ & $\begin{array}{c}0,94 \\
(50)\end{array}$ & $\begin{array}{l}15,00 \\
(450)\end{array}$ & $\begin{array}{c}46,10 \\
(900)\end{array}$ \\
\hline
\end{tabular}

Ag: Avicennia germinans; As: Avicennia schaueriana; Lr: Laguncularia racemosa; Rm: Rhizophora mangle. Valores entre parênteses representam a densidade de indivíduos por hectare. 
Tabela 6. Dominância em área basal (\%) e densidade relativa (\%) de indivíduos vivos e mortos por espécie nos sítios analisados no manguezal do estuário do rio São Mateus, ES, Brasil.

\begin{tabular}{|c|c|c|c|c|c|c|c|c|}
\hline \multirow[t]{2}{*}{ Sítio } & \multicolumn{4}{|c|}{ Vivos } & \multicolumn{4}{|c|}{ Mortos } \\
\hline & $\mathrm{Ag}$ & As & $\mathrm{Lr}$ & $\mathrm{Rm}$ & $\mathrm{Ag}$ & As & $\mathrm{Lr}$ & $\mathrm{Rm}$ \\
\hline S-1 & - & $\begin{array}{c}16,7 \\
(10,2)\end{array}$ & $\begin{array}{c}16,7 \\
(25,4)\end{array}$ & $\begin{array}{c}66,0 \\
(62,7)\end{array}$ & - & - & $\begin{array}{c}0,6 \\
(1,7)\end{array}$ & - \\
\hline S-2 & - & $\begin{array}{c}5,9 \\
(17,3)\end{array}$ & $\begin{array}{l}10,5 \\
(5,8)\end{array}$ & $\begin{array}{c}81,9 \\
(71,1)\end{array}$ & - & - & $\begin{array}{c}1,3 \\
(3,9)\end{array}$ & $\begin{array}{r}0,4 \\
(1,9)\end{array}$ \\
\hline S-3 & - & - & $\begin{array}{c}48,1 \\
(59,0)\end{array}$ & $\begin{array}{c}13,1 \\
(13,1)\end{array}$ & - & - & $\begin{array}{c}38,8 \\
(27,9)\end{array}$ & - \\
\hline S-4 & $\begin{array}{c}65,4 \\
(36,1)\end{array}$ & - & $\begin{array}{c}1,3 \\
(5,6)\end{array}$ & $\begin{array}{c}0,8 \\
(8,3)\end{array}$ & $\begin{array}{c}29,9 \\
(38,8)\end{array}$ & - & $\begin{array}{c}0,5 \\
(5,6)\end{array}$ & $\begin{array}{c}2,1 \\
(5,6)\end{array}$ \\
\hline
\end{tabular}

Ag: Avicennia germinans; As: Avicennia schaueriana; Lr: Laguncularia racemosa; Rm: Rhizophora mangle. Valores entre parênteses representam a densidade relativa de indivíduos.

(1980), o manguezal do rio São Mateus apresentaria os tipos franja, ribeirinho e bacia. O bosque do tipo bacia desenvolve-se em áreas condicionadas por uma suave depressão, onde a freqüência de inundação pelas marés é menor do que nos dois tipos anteriores. Como no presente estudo todas as parcelas foram posicionadas a $10 \mathrm{~m}$ da margem, não foi registrada a ocorrência deste tipo fisiográfico. O bosque do Sítio 1 é do tipo franja, pois ocorre na borda de um ambiente em contato com o mar, sendo menor a recepção de nutrientes quando comparado ao tipo ribeirinho. Os demais sítios são do tipo fisiográfico ribeirinho, ocorrendo ao longo do rio, sofrendo influência da maré e maior aporte de nutrientes.

De acordo com Cintrón et al. (1980), o bosque tipo franja tende a ter menor desenvolvimento estrutural quando comparado ao ribeirinho. No entanto, nos sítios 1 (franja) e 2 (ribeirinho), os manguezais são estruturalmente muito semelhantes e constituídos por uma espécie dominante absoluta ( $R$. mangle), em relação às outras (L. racemosa e A. schaueriana). A estrutura vegetal menos desenvolvida desses sítios responde aos maiores valores de salinidade da água intersticial e à maior hidrodinâmica local, decorrente da inundação do manguezal pelas águas do rio e das marés. Os sítios 3 (ribeirinho) e 4 (ribeirinho) apresentaram maior desenvolvimento estrutural, com elevada contribuição em área basal de indivíduos na classe de DAP $\geq 10,0 \mathrm{~cm}$ (S-3, $81 \%$ e S-4, 98\%), refletindo condições ambientais mais favoráveis.

O manguezal do rio São Mateus exibe fisionomia alterada por tensores naturais e antrópicos, tendo como agentes principais: erosão, assoreamento, desmatamento, invasão por palafitas, abertura de estradas, urbanização, lançamento de efluente doméstico e pesca predatória. O termo tensor é usado para descrever qualquer ação ou influência que retarde ou restrinja o funcionamento normal ou desenvolvimento de unidades biológicas. A ação contínua de um tensor constitui um dreno constante de energia e impede que o ecossistema atinja altos níveis de desenvolvimento (Lugo \& Snedaker 1974).

O gênero Avicennia é considerado o mais tolerante ao estresse ambiental (Cintrón-Molero \& SchaefferNovelli 1992). Pode ser encontrado dominando substratos altamente salinos ou em áreas sujeitas a baixas temperaturas, no entanto, é capaz de atingir melhor desenvolvimento estrutural em baixas salinidades, em locais livres de distúrbios ambientais induzidos pelo homem. No manguezal do rio São Mateus, A. germinans encontra condições bastante favoráveis para um bom desenvolvimento estrutural no Sítio 4, apresentando elevados valores para altura, DAP médio e área basal.

Em estudo anterior (C.C. Vale, dados não publicados) realizado apenas na desembocadura do rio São Mateus, R. mangle foi a espécie dominante em área basal (59\%), seguida de L. racemosa $(30 \%)$ e A. schaueriana (11\%). No presente estudo, foi confirmada a dominância de $R$. mangle $(66 \%)$, seguida de L. racemosa $(17 \%)$ e A. schaueriana (17\%). Como pode-se observar na Tab. 7, o desenvolvimento estrutural dos bosques em estudo apresenta-se bom,

Tabela 7. Variação na estrutura da vegetação em manguezais do Estado do Espírito Santo, ES, Brasil, de acordo com outros estudos.

\begin{tabular}{lccccc}
\hline Local & $\begin{array}{c}\text { Altura média } \\
(\mathrm{m})\end{array}$ & $\begin{array}{c}\text { DAP médio } \\
(\mathrm{cm})\end{array}$ & $\begin{array}{c}\text { Área basal } \\
\left(\mathrm{m}^{2} . \mathrm{ha}^{-1}\right)\end{array}$ & $\begin{array}{c}\text { Densidade } \\
\left(\text { ind.ha }^{-1}\right)\end{array}$ & Fonte \\
\hline Vitória & $5,23-17,30$ & $4,23-18,90$ & $5,39-29,80$ & $200-5.300$ & Carmo et al. 1995 \\
Vitória & 6,00 & $6,34-9,97$ & $13,90-17,70$ & $2.266-4.666$ & Carmo et al. 2000 \\
Vitória & $4,37-9,62$ & $7,89-13,60$ & $4,87-10,70$ & $733-996$ & Carmo et al. 1998a \\
Fundão & $3,76-5,10$ & $7,20-10,70$ & $9,35-17,90$ & $2.000-2.300$ & Carmo et al. 1998b \\
Este estudo & $5,40-12,00$ & $8,12-29,60$ & $7,21-31,10$ & $450-1.450$ & \\
\hline
\end{tabular}


quando comparado a outros manguezais do Estado do Espírito Santo.

A variabilidade na composição específica, assim como o acúmulo de biomassa, caracterizado pelo desenvolvimento estrutural, demonstram a grande maleabilidade dos manguezais e sua potencialidade de acomodação diante de marcadas diferenças ambientais entre pontos geograficamente próximos, tais como a erosão e a sedimentação aceleradas, a hidrodinâmica e o aporte de água doce. Essas diferenças devem ser levadas em consideração quando da proposta de planos de manejo, uma vez que cada manguezal exige estratégias peculiares para sua manutenção (Schaeffer-Novelli et al. 1994).

\section{Agradecimentos}

Ao Dr. Marcelo Trindade do Nascimento, pela revisão do manuscrito; ao Instituto Capixaba de Pesquisa, Assistência e Extensão Rural (INCAPER), Linhares, pelas análises do sedimento; à Polícia Ambiental de São Mateus e à Prefeitura Municipal de Conceição da Barra, pelo apoio relativo à embarcação. As autoras Elaine Bernini e Maria Amélia Bonfante Silva agradecem ao PIBIC/UFES, pela concessão de bolsa de iniciação à pesquisa.

\section{Referências bibliográficas}

Carmo, T.M.S.; Brito-Abaurre, M.G.; Senna-Melo, R.M.; Zanotti-Xavier, S.; Costa, M.B. \& Horta, M.M.M. 1995. Os manguezais da Baía Norte de Vitória, Espírito Santo: um ecossistema ameaçado. Revista Brasileira de Biologia 55(4): 801-808.

Carmo, T.M.S.; Almeida, R.; Oliveira, A.R. \& Xavier, S.Z. 1998a. Caracterização de um trecho do manguezal do rio da Passagem, Baía de Vitória, Vitória, ES, Brasil. Pp. 6-16. In: Anais do IV Simpósio de Ecossistemas Brasileiros. São Paulo, ACIESP, v. I.

Carmo, T.M.S.; Góes, P.; Almeida, A.P.L.S.; Sampaio, F.D.F. \& Assis, A.M. 1998b. Caracterização do manguezal do rio Reis Magos, Fundão, Espírito Santo. Pp.17-29. In: Anais do IV Simpósio de Ecossistemas Brasileiros. São Paulo, ACIESP, v. I.

Carmo, T.M.S.; Sforza, R.; Rocha, G.B.; Moreira, L.M.P. \& Silva, R.C.D. 2000. Caracterização da estrutura do manguezal de Goiabeiras Velha, Vitória, Espírito Santo. Pp.381-388. In: Anais do V Simpósio de Ecossistemas Brasileiros. São Paulo, ACIESP, v. II.
Cintrón, G.; Lugo, A.E.; Pool, D.J. \& Morris, G. 1978. Mangroves of arid environments in Puerto Rico and Adjacents Inlands. Biotropica 10(2): 110-121.

Cintrón, G.; Lugo, A.E. \& Martinez, R. 1980. Structural and functional properties of mangrove forests. Pp. 53-67. In: Annals of the Symposium Signaling the Complexion of the Flora of Panama. Panama, University of Panama.

Cintrón-Molero, G. \& Schaeffer-Novelli, Y. 1992. Ecology and management New World mangroves. Pp. 233-258. In: U. Seeliger (ed.). Coastal plant communities of Latin America. San Diego, Academic Press.

EMBRAPA, Centro Nacional de Pesquisa de Solos. 1997. Manual de métodos de análise do solo. Rio de Janeiro, EMBRAPA-CNPS.

Field, C. 1996. La restauración de ecosistemas de manglar. Japón, Sociedad Internacional para Ecosistemas de Manglar, Okinawa.

Lugo, A.E. \& Snedaker, S.C. 1974. The ecology of mangroves. Annual Review of Ecology and Systematic 5: 39-64.

Odum, W.E. \& Johannes, R.E. 1975. The response of mangrove to man-induced environmental stress. Pp. 52-62. In: E.J.F. Wood \& R.E. Johannes (eds.). Tropical Marine Pollution. Amsterdam, Elsevier Oceanography Series.

Schaeffer-Novelli, Y. 1995. Manguezal: ecossistema entre a terra e o mar. São Paulo, Caribbean Ecological Research.

Schaeffer-Novelli, Y. \& Cintrón, G. 1986. Guia para estudo de áreas de manguezal: estrutura, função e flora. São Paulo, Caribbean Ecological Research.

Schaeffer-Novelli, Y. \& Cintrón-Molero, G. 1994. Manguezais brasileiros: uma síntese sobre aspectos históricos (séculos XVI a XIX), zonação, estrutura e impactos ambientais. Pp. 333-341. In: Anais do Simpósio de Ecossistemas da Costa Brasileira. Subsídios a um gerenciamento ambiental. São Paulo, ACIESP, v. I.

Schaeffer-Novelli, Y.; Cintrón-Molero, G.; Adaime, R.R. \& Camargo, T.M. 1990. Variability of mangrove ecosystems along the Brazilian coast. Estuaries 13(2): 204-218.

Soares, M.L.G. 1999. Estrutura vegetal e grau de perturbação dos manguezais da Lagoa da Tijuca, Rio de Janeiro, RJ, Brasil. Revista Brasileira de Biologia 59(3): 503-515.

Suguio, K. 1973. Introdução a Sedimentologia. São Paulo, Edgar Blucher, EDUSP.

Vale, C.C. \& Ferreira, R.D. 1998. Os manguezais do litoral do Estado do Espírito Santo. Pp. 88-94. In: Anais do Simpósio de Ecossistemas da Costa Brasileira. São Paulo, ACIESP, v. I.

Walsh, G.E. 1974. Mangrove forests: a review. In: R.J. Reinold \& W.H. Queen (eds.). Ecology of Halophytes. New York, Academic Press. 